\title{
Cost-effectiveness of adjuvant chemotherapy for curatively resected gastric cancer with S-1
}

\author{
Akinori Hisashige $^{1 *}$, Mitsuru Sasako ${ }^{2}$ and Toshifusa Nakajima ${ }^{3}$
}

\begin{abstract}
Background: The effectiveness of specific regimens of adjuvant therapy for gastric cancer has not been verified by large clinical trials. Recently, several large trials attempted to verify the effectiveness of adjuvant therapy. The Adjuvant Chemotherapy Trial of TS-1 for Gastric Cancer in Japan, a randomized controlled trial of adjuvant S-1 therapy for resected gastric cancer, demonstrated significant improvement in overall and relapse-free survival, compared to surgery alone. To evaluate value for money of S-1 therapy, cost-effective analysis was carried out.

Methods: The analysis was carried out from a payer's perspective. As an economic measure, cost per quality-adjusted life-year (QALY) gained was estimated. Overall survival was estimated by the Kaplan-Meier method, up to 5-year observation. Beyond this period, it was simulated by the modified Boag model. Utility score is derived from interviews with sampled patients using a time trade-off method. Costs were estimated from trial data during observation, while in the period beyond observation they were estimated using simulation results. To explore uncertainty of the results, qualitative and stochastic sensitivity analyses were done.

Results: Adjuvant S-1 therapy gained 1.24 QALYs per patient and increased costs by $\$ 3,722$ per patient for over lifetime (3\% discount rate for both effect and costs). The incremental cost-effectiveness ratio (95\% confidence intervals) for over lifetime was estimated to be $\$ 3,016(\$ 1,441, \$ 8,840)$ per QALY. The sensitivity analyses showed the robustness of these results.
\end{abstract}

Conclusion: Adjuvant S-1 therapy for curatively resected gastric cancer is likely cost-effective. This therapy can be accepted for wide use in Japan.

Keywords: Chemotherapy, S-1, Adjuvant therapy, Gastric cancer, Cost-effectiveness, Quality-adjusted life-year

\section{Background}

Gastric cancer is a major health problem worldwide. It ranks second in all causes of death from cancer, with about 700,000 confirmed deaths annually [1,2]. In Japan, although its mortality ranks also second and has decreased in recent years, it still has the highest incidence despite advances in prevention and treatment [3]. While the internationally accepted standard treatment for patients with potentially resectable disease was surgery alone [4,5], meta-analyses of adjuvant chemotherapy for gastric cancer during the last few decades have shown reductions in mortality up to $18 \%$ [6,7]. However, these reductions were considered insufficient to change clinical practice.

\footnotetext{
* Correspondence: akih@k3.dion.ne.jp

${ }^{1}$ The Institute of Healthcare Technology Assessment, 2-24-10, Shomachi, 770-0044, Tokushima, Japan

Full list of author information is available at the end of the article
}

Recently, the effectiveness of specific regimens for resectable gastric and/or gastroesophageal cancer has been verified in large clinical trials. The chemoradiation therapy (INT-0116) in the US in 2001 [8], the perioperative chemotherapy (MAGIC) in Europe in 2006 [9], and the postoperative chemotherapy (ACTS-GC) in Japan in $2007[10,11]$ improved significantly overall survival (OS), and relapse-free survival (RFS) or progression-free survival (PFS), compared to surgery alone.

These studies have led to a new phase in the treatment of gastric cancer, even though there are several issues under discussion concerning them $[5,12,13]$. Postoperative chemoradiotherapy, perioperative triplet-chemotherapy, and postoperative S-1 mono-chemotherapy are now the standard therapies in the US, Europe and Japan, respectively $[5,12]$. Also, the status of adjuvant treatment of gastric

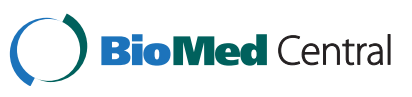


cancer has been evolving to improve and optimize the current standard of care across national boundaries.

Under these circumstances, from a perspective of healthcare policy, in choosing the best treatment among the different options available, clinical benefits of treatments should be balanced against the effects on costs, since rapid growth in healthcare expenditures creates an unsustainable burden. However, economic evaluation of adjuvant therapy for gastric cancer has been greatly lacking.

Our objective was to estimate the cost-effectiveness of adjuvant S-1 therapy in Japan. This study would provide basic information on the cost-effectiveness of adjuvant therapy for gastric cancer in Japan.

\section{Methods}

\section{Analytical overview}

Economic analysis was conducted retrospectively based on the ACTS-GC (ClinicalTrials.gov number, NCT00152217) $[10,11]$. Patients with completely resected stage II/III gastric cancer, who underwent gastrectomy with extended (D2) lymph-node dissection, were randomly assigned to either oral S-1 (40 mg/m² per day) for 1 year after surgery $(n=529)$ or surgery alone $(n=530)$. S-1 is an orally active combination of tegafur, gimeracil, and ostracil in a molar ratio of 1:0.4:1.

As a type of economic analysis [14], a cost-effective analysis was performed. Incremental costs and effectiveness of adjuvant S-1 therapy compared to surgery alone were evaluated. According to the effectiveness measure used (i.e., life-years (LYs) gained and quality-adjusted life-years (QALYs) gained), incremental cost-effectiveness ratios (ICERs) were calculated. In addition, confidence intervals of ICER were also estimated using the non-parametric bootstrap method [14].

The payer of National Health Insurance in Japan was adopted as a perspective of economic analysis [14]. Therefore, for costs, direct medical care costs (e.g., costs of tests, drugs, health care personnel, etc.) were examined, whereas indirect costs (e.g., time costs or production loss among patients and their families) were not considered. As a time horizon for evaluation, three levels of time periods (i.e., observational period [5 years], 10year follow-up and over lifetime) were considered. As the base case analysis, over lifetime was used, since this period covered long-term consequences of treatment on health and costs.

\section{Effectiveness}

The results of the ACTS-GC were used as evidence of effectiveness in the economic analysis. The clinical results have been presented in detail elsewhere [10,11]. As is shown in Table 1, between the S-1 therapy group and the surgery alone group, no statistical differences were
Table 1 Characteristics of subjects and clinical outcomes

\begin{tabular}{|c|c|c|}
\hline & S-1 therapy & Surgery alone \\
\hline Number of patients & 529 & 530 \\
\hline Age (median) & 63 & 63 \\
\hline Sex (male) & 367 & 369 \\
\hline \multicolumn{3}{|l|}{ Cancer stage (TNM classification) } \\
\hline IB & 1 & 0 \\
\hline$\|$ & 264 & 282 \\
\hline$\| \mathrm{A}$ & 170 & 157 \\
\hline$\| \mathrm{II}$ & 54 & 56 \\
\hline IV & 40 & 35 \\
\hline \multicolumn{3}{|l|}{ Type of lymph -node dissection } \\
\hline D1 & 0 & 1 \\
\hline D2 & 501 & 497 \\
\hline D3 & 28 & 32 \\
\hline \multicolumn{3}{|l|}{ Type of gastrectomy } \\
\hline Total & 220 & 201 \\
\hline Distal & 301 & 316 \\
\hline Proximal & 4 & 11 \\
\hline Other & 4 & 2 \\
\hline Adverse events more than grade $3^{*}$ & 155 & 80 \\
\hline \multirow[t]{2}{*}{ Total no. of relapses } & 162 & 221 \\
\hline & $\%$ & $\%$ \\
\hline 5-year survival $(95 \% \mathrm{Cl})$ & $72(68-76)$ & $61(57-65)$ \\
\hline 5-year relapse-free survival (95\% Cl) & $65(61-70)$ & $53(49-57)$ \\
\hline
\end{tabular}

The results are presented according to ITT (intention to treat): ${ }^{*}$ The results from the safety analysis.

observed in age, sex, pathological tumor stage, or type of lymph-node dissection and gastrectomy. The incidence of adverse events more than grade 3 in the S-1 therapy group was significantly higher than that in the surgery alone group. The OS and RFS rates in the S-1 therapy group were significantly higher than those in the surgery alone group $[10,11]$

Using patients' data, OS and RFS were estimated by the Kaplan-Meier method, up to 5 years from randomization. Beyond the observation period of 5 years, OS was simulated using the Boag model [15] combined with the independent competing risk model $[16,17]$ (Figure 1). While there is no explicit standard for extrapolation beyond the observation [18], this model showed an extreme goodness of fit, validated by observational data [17].

In this model, OS curve was decomposed into two components: the disease-specific survival curve and the disease-independent survival curve. In the first curve, only disease-specific (i.e., gastric cancer) deaths were counted as events, and all other deaths were censored; the converse applies to the second curve. The disease- 

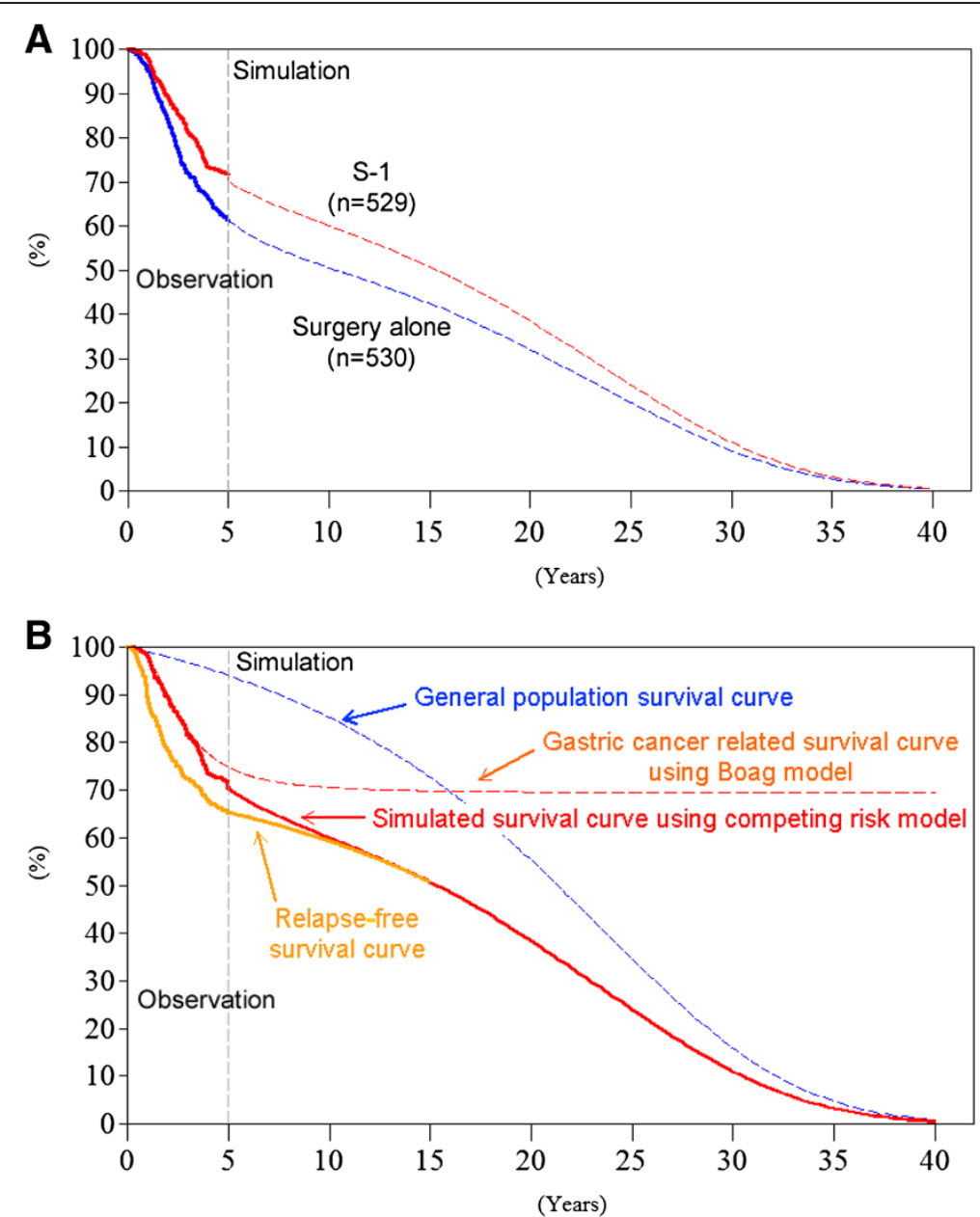

Figure 1 Survival curve and extrapolated survival estimate. (A) Survival curve in the S-1 and the control groups, (B) Survival curves using Boag and competing risk models and relapse-free survival curve in the S-1 group.

specific survival curve was then fitted by the Boag parametric model. As death from disease becomes rarer with increasing time, the disease-related survival curve approximates to a plateau (Figure 1B, gastric cancer related survival curve using the Boag model).

Instead of the original log-normal model, the loglogistic model was adopted in this analysis, according to the analysis of observational data of this trial. This loglogistic model was also supported by the analysis of a large database for gastric cancer in Japan [19]. In selecting a model among log-logistic, log-normal and Weibull models, Akaike's Information Criteria (AIC) were used [20].

The second curve, disease-independent curve was simulated by the survival curve of the general population matched for age and sex of the subjects, using national life tables (Figure 1B, general population survival curve).

The two simulated curves were then extended over lifetime and were recombined (multiplied) into a complete overall survival curve, using the competing risk model
(Figure 1B, simulated survival curve using competing risk model). Under the competing risk model, the simulated survival rate is simply derived from multiplying the disease-related survival rate by the disease-independent survival rate. The life years were estimated as the area under the curve (AUC). The survival rate and variance were obtained by maximum likelihood estimation of the Boag parameters (i.e., the cure rate, the mean and standard deviation of log survival time). A detailed description of QALY calculation is presented in Appendix.

For RFS, the log-logistic model was also adopted, according to the analysis of observational data in the study $[10,11]$ and AIC (Figure 1B, relapse-free survival curve).

The mean number of LYs and relapse-free LYs for patients in each group was estimated as the area under the OS and RFS curves, respectively [21]. In addition, QALYs were calculated from OS and RFS by weighting each survival in each interval by a utility value for each 
possible important health state (i.e., remission after surgery and relapse). Utility values for these health states were derived from an interview with random samples of patients in remission after surgery $(\mathrm{n}=23)$ and consecutive patients with relapse $(n=21)$, with informed consent, by using a time trade-off method. No statistical difference was observed in key characteristics between these samples and the population subjects $[10,11]$. The mean (and S.D.) of the utility values for remission after surgery and for metastasis were $0.851(0.121)$, and 0.349 (0.208), respectively. When the risk of relapse has diminished, the change in utility value for remission after surgery would be considered to be the same as that of the general population. We applied the weighting by age for each year of follow-up, based on a population survey for quality of life in Japan [22].

The utility reduction associated with adverse events was adjusted through the method adopted by Aballea, et al [23]. The utilities for hospitalization and the adverse events with grade 4 were reduced by $50 \%$. Also, 23\%, $19 \%$ and $36 \%$ reduction were applied for nausea, vomiting and stomatitis, and diarrhea, respectively.

\section{Cost}

Costs incurred for resources used during trial and subsequent follow-up were estimated from trial data and their extrapolation. Resource utilization during trial and follow-up was derived from individual patient history data. Since observations on many patients are censored in a clinical trial, subsequent costs are unknown. To correct for censoring, the inverse probability weighting method [21] was applied during the observation period. Beyond the observation period, costs related to gastric cancer (i.e., those for recurrence and end-of-life) were estimated using the simulation results. Costs were estimated from the National Health Insurance perspective using the National Health Insurance reimbursement list and drug price for 2007 [24,25]. The costs of adverse events and a recurrence were estimated based on patients' records during observation. The chemotherapy for the majority of recurrence was implemented according to the first-line therapy in the Japanese guidelines [26].

As most health economic guidelines (e.g., the UK, Canada, Netherlands, Germany and the US) indicated, unrelated health care costs in the later years of life were not included in this analysis [14]. All costs were converted from Japanese yen to US dollars based on OECD purchasing power parity in $2007(\$ 1=\backslash 120)$ [27].

\section{Discount}

Discounting for the time value of money was applied to both costs and effectiveness. In the base case analysis, both costs and effectiveness accruing beyond 1 year were discounted to present values at a rate of $3 \%$, following the recommendations of the US Panel on CostEffectiveness in Health and Medicine [28]. However, currently, much debate still surrounds two major points: the underlying discounting model and the differential discount rate for health and cost [28-30]. Therefore, the impact of discounting on the results was examined extensively by sensitivity analysis.

\section{Sensitivity analysis}

The uncertainty of the results was explored by stochastic and qualitative sensitivity analyses of important factors $[14,31,32]$. The impact of uncertainty on the estimated ICER due to the stochastic nature of sampled data was analyzed by applying a non-parametric bootstrap resampling technique (i.e., 5000 times) to both costs and effectiveness. Also, cost-effectiveness acceptability curve (CEAC) and net monetary benefit (NMB) analyses $[31,32]$ were performed. A number of qualitative oneway and two-way sensitivity analyses were conducted to explore the impact of alternative parametric assumptions on the results. These included alternative assumptions concerning time horizon, key cost parameter, recurrence rate, utility value, discount rate and simulation method. Also, the exclusion of end-of-life costs due to gastric cancer was examined by a sensitivity analysis, under the assumption that they may be considered as unrelated healthcare costs.

\section{Results}

\section{Effectiveness}

The mean QALYs (3\% discount rate) in each group are shown in Table 2. For 5-year observation, 10-year follow-up and over lifetime, the mean QALYs per patient for adjuvant S-1 therapy were 3.11, 5.08 and 8.65, respectively. Those for surgery alone were $2.84,4.45$ and 7.41, respectively. Adjuvant S-1 therapy gained 0.27, 0.64 and 1.24 QALYs per patient, for each period, respectively $(\mathrm{p}<0.05)$. The difference in QALYs was relatively smaller than that in LYs for 10-year follow-up and over lifetime.

\section{Cost}

The mean costs (no discounting) per patient in each group for the 5-year observation are shown in Table 3. The mean total cost per patient was $\$ 11,103$ in the S-1 therapy group, and $\$ 7,761$ in the surgery alone group. The costs of recurrence and end-of-life were the major component in both groups. Although S-1 therapy added over $\$ 4,000$ per patient to the ingredient cost of surgery alone, this was partly offset by the reduction of costs in recurrence and end-of-life of gastric cancer. As is shown in Table 2, for 5-year observation, 10-year follow-up and over lifetime, adjuvant S-1 therapy increased costs $(3 \%$ 
Table 2 Incremental effectiveness and costs of adjuvant S-1 therapy (discount rate: $3 \%$ for both effectiveness and costs)

\begin{tabular}{|c|c|c|c|}
\hline Period & $\begin{array}{c}\text { S-1 } \\
\text { therapy }\end{array}$ & $\begin{array}{l}\text { Surgery } \\
\text { alone }\end{array}$ & $\begin{array}{l}\text { Incremental effectiveness and } \\
\text { costs }(95 \% \mathrm{Cl})\end{array}$ \\
\hline \multicolumn{4}{|l|}{ Effectiveness } \\
\hline \multicolumn{4}{|c|}{ QALYS } \\
\hline $\begin{array}{l}\text { 5-year } \\
\text { observation }\end{array}$ & 3.11 & 2.84 & $0.27(0.11-0.42)$ \\
\hline $\begin{array}{l}\text { 10-year } \\
\text { follow-up }\end{array}$ & 5.08 & 4.45 & $0.64(0.28-0.99)$ \\
\hline Over lifetime & 8.65 & 7.41 & $1.24(0.48-1.96)$ \\
\hline \multicolumn{4}{|c|}{ Costs $(\$)$} \\
\hline $\begin{array}{l}\text { 5-year } \\
\text { observation }\end{array}$ & 10,802 & 7,408 & $3,389(2,616-4,174)$ \\
\hline $\begin{array}{l}\text { 10-year } \\
\text { follow-up }\end{array}$ & 12,110 & 8,523 & $3,585(2,750-4,411)$ \\
\hline Over lifetime & 13,057 & 9,346 & $3,722(2,911-4,512)$ \\
\hline \multicolumn{4}{|c|}{ Incremental cost-effectiveness ratio } \\
\hline & \multicolumn{2}{|c|}{$\begin{array}{l}\text { Cost (\$) per QALY } \\
\text { gained }\end{array}$} & $(95 \% \mathrm{Cl})$ \\
\hline $\begin{array}{l}\text { 5-year } \\
\text { observation }\end{array}$ & \multicolumn{2}{|c|}{12,716} & $(6,428-34,018)$ \\
\hline $\begin{array}{l}\text { 10-year } \\
\text { follow-up }\end{array}$ & \multicolumn{2}{|c|}{5,608} & $(2,855-14,569)$ \\
\hline Over lifetime & \multicolumn{2}{|c|}{3,016} & $(1,441-8,840)$ \\
\hline
\end{tabular}

$\mathrm{Cl}=$ confidence interval; QALYs = quality-adjusted life-years.

discount rate) per patient by $\$ 3,389, \$ 3,585$ and $\$ 3,722$ respectively, compared to surgery alone $(\mathrm{p}<0.05)$.

\section{Incremental cost-effectiveness ratio}

As is shown in Table 2, as the base case, the ICER (95\% confidence intervals) for over lifetime was estimated to be $\$ 3,016(\$ 1,441, \$ 8,840)$ per QALY, using the bootstrap method (3\% discount rate for both effect and cost). Those for 5-year observation and 10-year follow-up were $\$ 12,716$ and $\$ 5,608$ per QALY, respectively. There is little difference between costs per LY gained and costs per QALY gained.

\section{Sensitivity analysis}

The results of probabilistic sensitivity analyses are shown in Figures 2. Figure 2A shows ICER (cost per QALY gained) scatter plots based on 5,000 samples. All points resided in the northeast quadrant (i.e., more effective and more costly). All points were located under the diagonal line indicating the ICER of $\$ 50,000$ per QALY gained. The CEAC is presented in Figure $2 \mathrm{~B}$. If the value of an additional QALY was $\$ 6,220$, the likelihood of S-1 therapy being cost-effective was $95 \%$. The NMB curve is shown in Figure $2 \mathrm{C}$. The value of an additional QALY was $\$ 3,016$, when the NMB curve crossed the horizontal axis.
Table 3 Mean costs per patient during observation period (no discounting)

\begin{tabular}{|c|c|c|c|c|}
\hline \multirow{3}{*}{$\begin{array}{l}\text { Item } \\
\text { Unit cost }(\$)\end{array}$} & \multicolumn{2}{|c|}{ S-1 therapy } & \multicolumn{2}{|c|}{ Surgery alone } \\
\hline & Quantity & $\overline{\text { Cost }(\$)}$ & Quantity & Cost (\$) \\
\hline & \multicolumn{2}{|c|}{ (No. of units) } & \multicolumn{2}{|c|}{ (No. of units) } \\
\hline \multicolumn{5}{|l|}{ Consultation } \\
\hline Outpatient: 5.8 & 22.4 & 131 & 8.3 & 49 \\
\hline \multicolumn{5}{|l|}{ Treatment } \\
\hline S-1 drug (mg): 0.3 & 15,156 & 4,367 & NA & \\
\hline Prescription: 4.9 & 10.5 & 52 & NA & \\
\hline \multicolumn{5}{|l|}{ Tests } \\
\hline \multicolumn{5}{|l|}{ Imaging tests } \\
\hline CT: 124.3 & 5.1 & 629 & 4.9 & 608 \\
\hline Chest X-ray: 21.1 & 2.0 & 42 & 2.0 & 43 \\
\hline Echogram: 44.2 & 1.8 & 80 & 1.9 & 85 \\
\hline Endoscopy: 95.0 & 1.5 & 147 & 1.7 & 164 \\
\hline Others: 156.0 & 0.2 & 34 & 0.2 & 38 \\
\hline \multicolumn{5}{|l|}{ Laboratory tests } \\
\hline Blood test: 35.0 & 21.3 & 746 & 9.1 & 319 \\
\hline Tumor markers: 33.3 & 8.6 & 286 & 8.6 & 288 \\
\hline
\end{tabular}

Adverse effects

Anti-ulcerants: 6.0

Anti-biotics: 15.4

Anti-diarrhoeals: 5.4

Anti-emetics: 64.8

G-CSF: 106.9

Blood transfusion: 188.5

Recurrence

S-1: 3,694

Paclitaxel: 5,298

S-1 + cisplatin: 5,594

S-1 + paclitaxel: 5,247

5FU + methotrexate: 4,748

$\begin{array}{llll}0.3 & 2 & 0.2 & 1\end{array}$

$\begin{array}{lll}0.2 & 3 & 0.1\end{array}$

0.2

0.1

0.1

0.0

0.0

$$
0.0
$$

0.0

0

$\begin{array}{cccc}0.1 & 487 & 0.3 & 1,394 \\ 0.1 & 422 & 0.0 & 101 \\ 0.0 & 227 & 0.1 & 244 \\ 0.0 & 162 & 0.0 & 129 \\ 0.0 & 65 & 0.0 & 144 \\ 0.0 & 162 & 0.0 & 43 \\ 0.0 & 211 & 0.0 & 201\end{array}$

Others: 4,892

End of life

\begin{tabular}{llccc} 
Drugs/injections & 0.3 & 1,064 & 0.4 & 1,367 \\
Treatments & 0.3 & 233 & 0.4 & 321 \\
Operations/anesthesia & 0.3 & 213 & 0.4 & 296 \\
Diagnostic tests & 0.3 & 221 & 0.4 & 353 \\
Total costs per patient & \multicolumn{2}{c}{11,103} & \multicolumn{2}{c}{7,761} \\
(SD) & $(6,832)$ & $(6,787)$ \\
\hline
\end{tabular}

$\mathrm{NA}=$ not applicable.

A number of qualitative sensitivity analyses are shown in Tables 2 and 4. As to time horizon (Table 2), from 5year observation to over lifetime, ICER varied from $\$ 12,716$ to $\$ 3,016$, as mentioned before. 

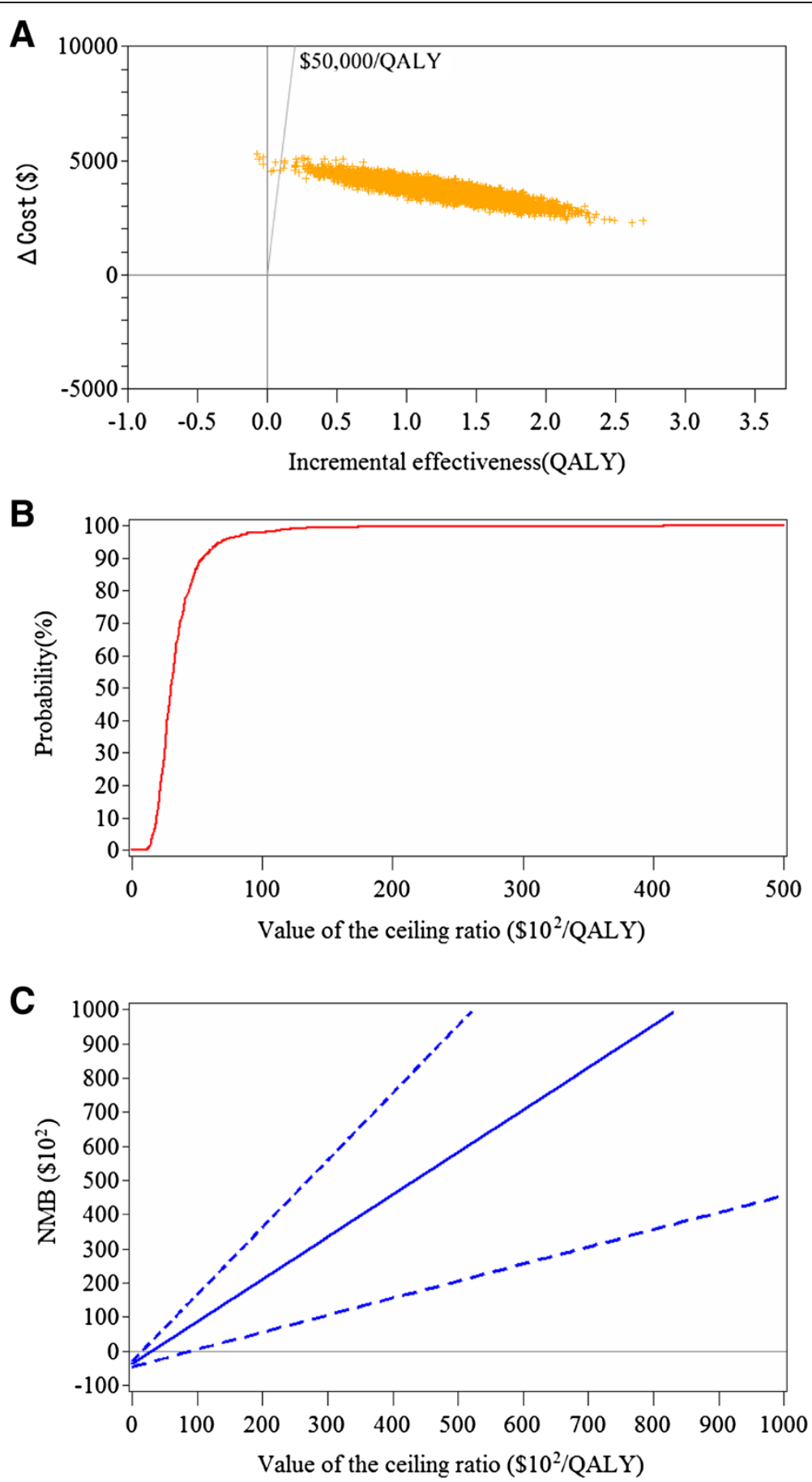

Figure 2 Stochastic sensitivity analyses. (A) Incremental cost-effectiveness scatter plot of adjuvant S-1 therapy, (B) Cost-effectiveness acceptability curve of adjuvant S-1 therapy, (C) Net monetary benefit curve of adjuvant S-1 therapy with 95\% confidence intervals.

The two-way sensitivity analysis of discount rate for both costs and effect showed a relatively small change in ICER. ICER was lowest (\$2,194/QALY) without discounting and highest (\$3,628/QALY) at the discount rate of $5 \%$ for both costs and effectiveness. ICER increased with increase in discount rate of both cost and effect.
The results of one-way sensitivity analyses are shown in Table 4. Variations in recurrence rate, utility value, QALYs, the acquisition cost of S-1, recurrence cost, endof-life cost, and simulation model did not greatly change ICER. With variations of these variables, ICERs varied from $\$ 1,901$ to $\$ 7,696$ per QALY gained. 
Table 4 One-way sensitivity analysis of important factors

\begin{tabular}{|c|c|}
\hline Factor & $\begin{array}{l}\text { Cost-effectiveness ratio } \\
\text { (\$/QALY gained) }\end{array}$ \\
\hline Base case analysis & 3,016 \\
\hline \multicolumn{2}{|l|}{ Simulation model } \\
\hline Log-normal & 2,874 \\
\hline Weibull & 3,341 \\
\hline $\begin{array}{l}\text { Recurrence rate } \\
\text { ( } 95 \% \text { Cl: } 30.5 \%-38.8 \%)\end{array}$ & $2,446-3,891$ \\
\hline \multicolumn{2}{|l|}{ Utility } \\
\hline $\begin{array}{l}\text { Remission after surgery } \\
\text { ( } 95 \% \mathrm{Cl}: 0.788-0.898 \text { ) }\end{array}$ & $2,825-3,231$ \\
\hline Metastasis (95\% Cl: 0.231 - 0.473) & $2,998-3,032$ \\
\hline QALY gained (95\% Cl: $0.48-1.96)$ & $1,901-7,696$ \\
\hline $\begin{array}{l}\text { Recurrence cost } \\
\text { ( } 95 \% \text { Cl: } \$ 2,032-\$ 2,422 \text { ) }\end{array}$ & $2,834-3,149$ \\
\hline $\begin{array}{l}\text { End of life cost } \\
(95 \% \text { Cl: } \$ 3,997-\$ 4,766)\end{array}$ & $2,682-3,302$ \\
\hline $\begin{array}{l}\text { Exclusion of end-of-life costs due to } \\
\text { gastric cancer }\end{array}$ & 3,677 \\
\hline S-1 cost $(95 \%$ Cl: $\$ 4,322-\$ 4,772)$ & $2,810-3,173$ \\
\hline $\begin{array}{l}\text { Total cost difference } \\
(95 \% \text { Cl: } \$ 2,911-\$ 4,512)\end{array}$ & $2,347-3,638$ \\
\hline
\end{tabular}

Discount rate: $3 \%$ for both cost and effectiveness, Period: lifetime.

\section{Discussion}

From the perspective of the National Health Insurance in Japan, this cost-effectiveness analysis showed that S-1 adjuvant therapy for gastric cancer gained LYs and QALYs, while it increased costs, compared with surgery alone (Table 2). The ICER of S-1 therapy can be ranked close to the top of the league table of cost-utility in oncology [33]. There is some consensus about the threshold of willingness to pay for additional QALY internationally (e.g., $\$ 50,000$ in the US, $£ 30,000$ in the UK, or AUS $\$ 42,000$ in Australia) [34]. A recent review suggested that the plausible threshold is $\$ 109,000 /$ QALY, rather than \$50,000/QALY [35]. In Japan, the social value (i.e., willingness to pay) for QALY gained was estimated to be from $\$ 53,000$ to $\$ 56,000$ by a nationwide mail survey using conjoint analysis [36]. Since the ICER of S-1 therapy is far below these thresholds, it is considered acceptable.

There has been little evidence on economic evaluation of adjuvant therapy for gastric cancer. A cost-effectiveness analysis evaluating postoperative chemoradiotherapy for gastric cancer in the US showed that the incremental costeffectiveness ratio was $\$ 38,400$ per QALY gained [37]. This ratio is 14 times higher and less efficient than that in our study, although several factors such as clinical practice patterns and relative costs should be considered in transferring evaluation data [14]. Moreover, since there is no genuine utility information in calculating QALY in the report [37], its validity and plausibility would be questionable.

The results of this study are subject to uncertainty and assumptions. To estimate stochastic uncertainty of ICER due to sampling variation or error, probabilistic sensitivity analyses $[14,31,32]$ were performed (Table 2, Figure 2). Cost-effectiveness scatter plots showed that all points of ICERs were located under the diagonal line indicating $\$ 50,000 / \mathrm{QALY}$. CEAC and NMB curves give more information. If a decision-maker was willing to pay $\$ 6,220$ to achieve an additional QALY, the likelihood of S-1 therapy being acceptable as cost-effective was 95\% (Figure $2 \mathrm{~B}$ ). The NMB curve shows that S-1 therapy was beneficial, if a decision-maker was willing to pay $\$ 2,782$ (Figure 2C). These values are extremely low compared with the thresholds (e.g., \$50,000).

The time horizon is an important issue to sufficiently capture relevant costs and health outcomes of S-1 adjuvant therapy. The observation period of the ACTS-GC, 5 years was limited. While most costs were incurred mainly in the observational period, LYs gained would continue after it. In this study, a simulation model was used to extrapolate its results. There is a variety of ways for simulation [18], but no uniform methodology available. We used the Boag model, which is indicated to be predictive for prognosis of gastric cancer [17]. In a sensitivity analysis, the ICER of the observational period was much higher than that of over lifetime (the base case), but it is very low compared with the thresholds. Also, the results of other simulation methods indicated similar results. The exclusion of end-of-life costs due to gastric cancer slightly increased the ICER, but it still remained far under the threshold (Table 4). These analyses show the robustness of this study.

The key drivers of cost-effectiveness results of S-1 are mainly the acquisition cost of S-1 and the costs related to recurrence and death. The S-1 therapy partly offset the acquisition cost of S-1 by the savings achieved by reduction of these costs. In one-way sensitivity analysis (Table 4), varying recurrence rates and costs of recurrence and end-of-lie did not have substantial impact on cost-effectiveness. Varying acquisition cost, which was the other cost driver, also did not have major impact on cost-effectiveness (Table 4). The sensitivity analysis of total cost corresponded with these results.

Cost-effectiveness analysis using QALYs offers the opportunity to consider both quantity and quality of survival. However, no substantial difference in ICERs was observed between cost per LY gained and QALY gained (Table 2). In this study, utility values were derived from a relatively small number of patients with gastric cancer, but this is the first study which directly evaluated the utilities among patients with gastric cancer. These values are similar to those observed for general cancer (i.e, 
0.89 after surgery and 0.44 for metastasis) in the Canadian survey among the general population [38]. The sensitivity analysis on range of utility values for remission after surgery and metastasis revealed no major change in cost-effectiveness (Table 4). In a sizable fraction of cost-effectiveness analyses, utility weighting was indicated not to substantially alter the estimated costeffectiveness of an intervention [39]. It is thus suggested that sensitivity analyses using ad hoc adjustment or weight from the literature may be sufficient. Our results support this conclusion.

The impact of discounting for the time value of money on the results was examined extensively by two-way sensitivity analysis. Although ICERs were more sensitive to effectiveness discounting than cost discounting, there was no substantial change in cost-effectiveness. The main reason is likely to be that major costs were incurred during the early phase of follow-up and improved survival continued for a relatively long time.

There are additional limitations in the analysis that should be commented on. First, the perspective of this analysis is that of a payer for healthcare, rather than a society. From a societal perspective, the range of costs is broader and includes other costs such as indirect costs. Since S-1 therapy increased OS and decreased recurrence, these factors would reduce indirect costs and decrease its ICER.

Second, the issue of generalizability of this study to other countries should be carefully examined. S-1 is widely used in Asian countries (e.g., Japan, Korea, Singapore and China). However, it is difficult to determine the relative effectiveness of $\mathrm{S}-1$, compared with the preoperative chemoradiotherapy in the US and the preoperative triplet-chemotherapy in Europe, since there is no direct comparison among them [8-10]. Moreover, there are several critical arguments around these studies. For example, the INT-0116 study attracted some criticism on the grounds of poor standardization of surgery and insufficient extended dissection of regional lymph nodes [5]. Thus it was argued that the chemoradiation component of the adjuvant treatment had compensated for less-than-ideal surgery. On the other hand, the quality of the MAGIC trial was pointed out to be much poorer than that of the INT-0116 study, in the areas of active quality control of surgery, data management, and compliance with protocol [12]. As to S-1, a difference in S-1 phamacokinetics was observed between Asians and Caucasians [13].

Recently, although the subjects did no have resectable gastric cancer like in this study, but advanced gastric cancer, the First-Line Advance Gastric Cancer Study (FLAGS) [40], a multinational trial, showed that cisplatin/S-1 was statistically non-inferior in overall mortality to cisplatin/5-FU and showed a significantly improved safety profile in Western countries. While S-1 is now approved by the EMEA in European countries, an international head-to-head comparison between S-1 therapy and the Western standard therapies will be required to confirm relative effectiveness and cost-effectiveness of S-1 therapy.

\section{Conclusion}

S-1 adjuvant therapy for gastric cancer gained LYs and QALYs, while it increased costs, compared with surgery alone. The ICER of S-1 therapy can be ranked close to the top of the league table of cost-utility in oncology and far below the social value or threshold for QALY gained in Japan. S-1 therapy for curatively resected gastric cancer is likely cost-effective. This therapy can be accepted for wide use in Japan.

\section{Appendix: the method of QALY calculation}

A.1 Calculation of QALY

QALYi (u), defined as the QALY at year i, was calculated by the following Equation (1), in which uNR represents the utility value of no relapse and $u R$ represents the utility value of relapse.

$$
\begin{aligned}
\operatorname{QALYi}(u)= & u N R \times \text { mean relapse-free rate }+u R \\
& \times(\text { mean survival rate-mean relapse-free rate })
\end{aligned}
$$

If $\mathrm{d}$ is the discount rate, the equation becomes $Q A L Y$ $(u)=\operatorname{Lid}(i-1) \times Q A L Y i(u)$.

The mean rate of survival was calculated as the area under the curve (AUC) of OS, and the mean rate of relapse-free survival was calculated as the AUC of RFS, using the trapezoidal approximation rule.

\section{A.2 Estimate of survival curves of lifetime OS}

When estimating the survival curves of lifetime OS, it was assumed that some patients in this study would be cured in response to treatment. This model is called the Boag (cure) model or mixture cure model. This statistical model assumes a mixed distribution of survival time among cured patients and uncured patients.

$\mathrm{Y}$ is defined as a variable indicating the presence or absence of cure in patients. $\mathrm{Y}=0$ stands for cure, and $\mathrm{Y}=1$ stands for non-cure. If $\mathrm{p}$ is defined as the probability of non-cure as represented by $\mathrm{p}=\operatorname{Pr}(\mathrm{Y}=1)$, and $\mathrm{T}$ is a random variable indicating the survival time, the $\mathrm{cu}$ mulative distribution function of $\mathrm{T}$ is represented by the following Equation (2).

$$
\begin{aligned}
F(t) & =\operatorname{Pr}(T \leq t) \\
& =p \cdot \operatorname{Pr}(T \leq t / Y=1)+(1-p) \cdot \operatorname{Pr}(T \leq t / Y=0)
\end{aligned}
$$

It was assumed that no events occur because of cure in cured patients. In other words, if $\operatorname{Pr}(\mathrm{T} \leq \mathrm{t} \mid \mathrm{Y}=0)=0$, the 
distribution function would be represented by Equation (2). This is referred to as a cure model.

$$
F(t)=p \cdot(t / Y=1)
$$

In the cure model, the probability density function $f(t)$ and survival function $\mathrm{S}(\mathrm{t})$ are represented by the following Equations (4).

$$
\begin{aligned}
& f(t)=p \cdot f(t / Y=1) \\
& S(t)=(1-p)+p \cdot S(t / Y=1)
\end{aligned}
$$

A logistic regression model was assumed to calculate the probability of non-cure $p$. In this model, $p$ is calculated by Equation (5), in which $\mathrm{z}$ is a covariance vector, $\mathrm{x}=(1, \mathrm{z})^{\prime}$ (' stands for vector transposition), and $\mathrm{b}$ is a regression coefficient vector of covariance.

$$
p(x)=\frac{\exp \left(b^{\prime} x\right)}{1+\exp \left(b^{\prime} x\right)}
$$

The Boag model [15] assumes a log-normal distribution for the survival time of uncured patients, but a loglogistic distribution was assumed in the present study. Furthermore, sensitivity analysis was also performed assuming a log-normal distribution and a Weibull distribution, and the maximum likelihood method was used to estimate the parameters using observational data of the ACTS-GC trial $[10,11]$. The goodness of fit of the model was evaluated with Akaike's information criteria (AIC). A log-logistic distribution has two parameters $\boldsymbol{\theta}=$ $(\gamma, \lambda)^{\prime}$, and the survivor function is as follows:

$$
S(t ; \theta)=\frac{1}{1+\lambda \cdot t^{\gamma}}
$$

The statistical software package SAS (version 9.2) was used to fit the data to the aforementioned models, and the probabilities of non-cure $(p)$ were estimated to be 0.306 and 0.422 in the $\mathrm{S}-1$ group and surgery alone group, respectively. The loglogistic distribution parameters $\lambda$ and $\gamma$ were 0.9724 and 0.4121 , respectively. The value of AIC for the log-logistic model was 1,678. Those for log-normal and Weibull models were 2,113 and 2,117, respectively. The programs used to estimate the model parameters were the SAS macro for survival models with a cured fraction (Mixture Cure Models).

To examine the validity of the log-logistic model, the distribution of survival time of cured patients was also analyzed using data on patients with gastric cancer obtained from the Cancer Institute Hospital (19462004), which has an open database [19]. The approach used was as follows: First, data on patients who met the following 6 eligibility criteria corresponding to the ACTS-GC trial $(n=1,457)$ were extracted from all data $(n=13,740)$. The median age of the patients extracted from the database was 57 years, which was 6 years younger than the median age of 63 years in the ACTSGC trial. Kaplan-Meier curves were plotted using the extracted patient data, defining only death from gastric cancer as an event. The curve reached a plateau after about 20 years (corresponding to an age of 77 years). These data were used for cure models assuming a Weibull distribution, log-normal distribution, and loglogistic distribution. The goodness of fit of the data as indicated by the AIC was best for the log-logistic distribution. While the value of AIC for the log-logistic model was 1,845 , those for the log-normal and Weibull models were 2,071 and 2,105, respectively.

Eligibility criteria of the ACTS-GC trial

1) A histologically confirmed diagnosis of gastric cancer

2) Lymph-node dissection of D2 or greater, with a curability of A or B

3) Stage II, IIIA, or IIIB disease

4) No liver metastasis, hematogenous metastasis, or distant metastasis

5) An age of 20 to 80 years

6) No previous treatment (chemotherapy, radiotherapy) received

Finally, the OS curve was constructed by combining the disease-specific survival curve (cure parametric model) and the disease-independent survival curve (the general population matched for age and sex of the subjects) based on the competing risk model. The actual calculation was done using a competitive risk model and the following Equation (7), in which $S_{B}(t)$ stands for the survival rate in the disease-specific survival curve (= cure model curve), $\mathrm{S}_{\mathrm{C}}(\mathrm{t})$ stands for the survival rate of the general population in the disease-independent survival curve, and $S_{A}(t)$ is the estimated rate of OS after the observation period. The structure of the OS curve was presented in Figure 1B.

$$
S_{A}(t)=S_{B}(t) S_{C}(t)
$$

\section{Competing interest}

MS reports receiving lectures fees from Taiho. All other authors: none to declare.

\section{Authors' contributions}

$\mathrm{AH}$ : study concept and design, acquisition of economic data, analysis and interpretation of economic data, and preparation of manuscript.MS, SN: acquisition of subjects and/or clinical data, analysis and interpretation of clinical data. All authors read and approved the final manuscript.

\section{Acknowledgement}

We thank Dr. Myles O'Brien, Prof. of Mie Prefectural College of Nursing, for his English editing. 


\section{Author details}

${ }^{1}$ The Institute of Healthcare Technology Assessment, 2-24-10, Shomachi, 770-0044, Tokushima, Japan. ${ }^{2}$ Department of Upper Gastrointestinal Surgery, Hyogo College of Medicine, 663-8501, Hyogo, Japan. ${ }^{3}$ Division of Surgery, The Cancer Institute Hospital, 135-8550, Tokyo, Japan

Received: 28 February 2013 Accepted: 26 September 2013 Published: 1 October 2013

\section{References}

1. Kamangar F, Dores GM, Anderson WF: Patterns of cancer incidence, mortality, and prevalence across five continents: defining priorities to reduce cancer disparities in different geographic regions of the world. J Clin Oncol 2006, 24:2137-2150.

2. Parkin DM, Bray F, Ferlay J, Pisani P: Global Cancer Statistics, 2002. CA Cancer J Clin 2005, 55:74-108.

3. Committee of Cancer Statistics: Cancer Statistics 2005. Tokyo: Foundation of Promotion of Cancer Research; 2008 (in Japanese).

4. Cunningham D, Chua YJ: East meets west in the treatment of gastric cancer. N Engl J Med 2007, 357:1863-1864.

5. Foukakis T, Lundell L, Gubanski M, Lind PA: Advances in the treatment of patients with gastric carcinoma. Acta Oncol 2007, 46:277-285.

6. Mari E, Floriani I, Tinazzi A, et al: Efficacy of adjuvant chemotherapy after curative resection for gastric cancer: a metaanalysis of published randomized trials. Ann Oncol 2000, 11:837-843.

7. Panzini I, Gianni L, Fattori PP, et al: Adjuvant chemotherapy in gastric cancer: a meta-analysis of randomized trials and a comparison with previous meta-analyses. Tumori 2002, 88:21-7.

8. Macdonald JS, Smalley SR, Benedetti J, et al: Chemoradiotherapy after surgery compared with surgery alone for adenocarcinoma of the stomach or gastroesophageal junction. N Engl J Med 2001, 345:725-30.

9. Cunningham D, Allum WH, Stenning SP, et al: Perioperative chemotherapy versus surgery alone for resectable gastroesophageal cancer. $N$ Engl Med 2006, 355:11-20.

10. Sakuramoto S, Sasako M, Yamaguchi T, et al: Adjuvant chemotherapy for gastric cancer with s-1, an oral fluoropyrimidine. N Engl J Med 2007, 357:1810-1820.

11. Sasako M, Sakuramoto S, Katai H, et al: Five-year outcomes of a randomized phase III trial comparing adjuvant chemotherapy with Sversus surgery alone in stage II or III gastric cancer. J Clin Oncol 2011, 29:4387-4393.

12. Sasako M: Surgery and adjuvant chemotherapy. Int J Clin Oncol 2008 13:193-195.

13. Lenz HJ, Lee FC, Haller DG, et al: Extended safety and efficacy data on S-1 plus cisplatin in patients with untreated, advanced gastric carcinoma in a multicenter phase II study. Cancer 2007, 109:33-40.

14. Drummond MF, Sculpher MJ, Torrance GW, et al: Methods for the Economic Evaluation of Health Care Programmes. 3rd edition. NY: Oxford Univ Press; 2005.

15. Boag JW: Maximum likelihood estimates of the proportion of patients cured by cancer therapy. J Roy Stat Soc 1949, B11:15-53.

16. Gross AJ, Clark VA: Survival distributions: Reliability applications in the biomedical sciences. NY: John Wiley \& Sons; 1975

17. Maetani S, Nakajima T, Nishikawa T: Parametric mean survival time analysis in gastric cancer patients. Med Dec Making 2004, 24:131-141.

18. Lee ET, Go OT: Survival analysis in public health research. Ann Rev Pub Health 1997, 18:105-134.

19. Nakajima S, Yamaguchi T: Database for gastric cancer in the Cancer Institute Hospital. Tokyo, Japan: Kanehara; 2006 (in Japanese).

20. Akaike $\mathrm{H}$ : A new look at the statistical model identification. IEEF Trans Autom Control 1974, 19:716-723.

21. Willan AR, Briggs AH: Statistical Analysis of Cost-Effectiveness Data. Chichester: John Wiley \& Sons; 2006

22. Hisashige A: Quality of life among Japanese general population, Economic evaluation of healthcare services by disease management, the Research on Health Science 2000, the Ministry of Health and Welfare. 2001. in Japanese.

23. Aballéa S, Chancellor JV, Raikou M, et al: Cost-effectiveness analysis of oxaliplatin compared with 5-fluorouracil/leucovorin in adjuvant treatment of stage III colon cancer in the US. Cancer 2007, 109:1082-1089.

24. Jiho: Encyclopedia of Drugs Listed for Insurance. Tokyo: Jiho Inc; 2007. in Japanese.
25. Institute of Social Insurance: Interpretation for Table of Points of Medical Practice. Tokyo: Institute of Social Insurance; 2007. in Japanese.

26. Japanese Gastric Cancer Association: Gastric cancer treatment guidelines. 2nd edition. Tokyo: Kanehara; 2004. in Japanese.

27. OECD: GDP PPPS and delivered indices for all OECD countries, OECD main economic indicators. Paris: OECD; 2008

28. Gold MR, Siegel JE, Russell LB, Weinstein MC: Cost-Effectiveness in Health and Medicine. NY: Oxford Univ Press; 1996.

29. Brouwer WB, Niessen LW, Postma MJ, Rutten FF: Need for differential discounting of costs and health effects in cost-effectiveness analyses. BMJ 2005, 331:446-448.

30. Bos JM, Postma MJ, Annemans L: Discounting health effects in pharmacoeconomics evaluations, current controversies. Pharmacoeconomics 2005, 23:639-649.

31. Briggs $\mathrm{AH}$ : Statistical approaches to handling uncertainty in health economic evaluation. Eur J Gastroenterol Hepatol 2004, 16:551-561.

32. Glick HA, Briggs AH, Polsky D: Quantifying stochastic uncertainty and presenting results of cost-effectiveness analyses. Expert Rev Pharmacoeconomics Outcome Res 2001, 1:89-100.

33. Earle CC, Chapman RH, Baker CS, et al: Systematic overview of cost-utility assessments in oncology. J Clin Oncol 2000, 18:3302-3317.

34. Jonsson B: Changing health environment: the challenge to demonstrate costeffectiveness of new compounds. Pharmacoeconomics 2004, 22(Suppl 4):5-10.

35. Braithwaite RS, Meltzer DO, King JT Jr, et al: What does the value of modern medicine say about the $\$ 50,000$ per quality-adjusted life-year decision rule? Med Care 2008, 46:349-356.

36. Ohkusa Y, Sugawara T: Research for willingness to pay for one QALY gain. Med Soc 2006, 16(2):157-165 (in Japanese).

37. Wang SJ, Fuller CD, Choi M, Thomas CR: A cost-effectiveness analysis of adjuvant chemoradiotherapy for resected gastric cancer. Gastrointest Cancer Res 2008, 2:57-63.

38. Evans WK, Garber SKC, Spence ST, Will ST: Health status descriptions for Canadians: Cancers. Ottawa: Statistics Canada; 2005.

39. Chapman $\mathrm{RH}$, Berger $\mathrm{M}$, Weinstein $\mathrm{MC}$, et al: When does quality-adjusted lifeyears matter in cost-effectiveness analysis? Health Econ 2004, 13:429-436

40. Ajani JA, Rodriquez W, Bodoky G, et al: Multicenter phase III comparison of cisplatin/S-1 with cisplatin/ infusional fluorouracil in patients with advanced gastric or gastroesophageal adenocarcinoma study: the FLAGS trial. J Clin Oncol 2010, 28:1547-53.

doi:10.1186/1471-2407-13-443

Cite this article as: Hisashige et al: Cost-effectiveness of adjuvant chemotherapy for curatively resected gastric cancer with S-1. BMC Cancer 2013 13:443.

\section{Submit your next manuscript to BioMed Central and take full advantage of:}

- Convenient online submission

- Thorough peer review

- No space constraints or color figure charges

- Immediate publication on acceptance

- Inclusion in PubMed, CAS, Scopus and Google Scholar

- Research which is freely available for redistribution

Submit your manuscript at www.biomedcentral.com/submit
C Biomed Central 